\title{
Development of a Cost-Effective Design for the Fusion Ignition Research Experiment
}

\author{
Philip J. Heitzenroeder* and the FIRE Engineering Team \\ *Princeton Plasma Physics Laboratory, PO Box 451, Princeton, NJ 08543 USA
}

\begin{abstract}
The Fusion Ignition Research Experiment (FIRE) is one of the components of a US Next Step Options (NSO) study which is considering what major experiments might be undertaken in a restructured US Fusion Sciences Program. FIRE is designed for a plasma current of $\sim 6.5 \mathrm{MA}$, a burn time of at least $10 \mathrm{~s}$, and $\mathrm{Q}$ in the range of 5 to 10 . FIRE has a major radius of $2.0 \mathrm{~m}$, a minor radius of $0.525 \mathrm{~m}$, and a field on axis of 10T. All of the coils are inertially cooled by liquid nitrogen. FIRE will operate primarily in a double null configuration with an $x$-point triangularity of 0.8 and an $x$ point elongation of 2.2. In addition to these technical requirements, a major goal for the FIRE project is for a total project cost of approximately \$1B (in FY 99 dollars). This paper describes the process and rationale for the engineering design chosen for FIRE, taking into account both the performance and cost goals.
\end{abstract}

Index terms-- FIRE; NSO; tokamak engineering;

\section{INTRODUCTION}

The Next Step Options (NSO) study has been organized as a national integrated physics / engineering design activity within the Virtual Laboratory for Technology which is headed by Charles Baker at UCSD. The NSO study is led by John Schmidt of the Princeton Plasma Physics Laboratory. The first step in the NSO study was to formulate a preliminary set of goals and requirements for the development of an optimized Burning Plasma Strategy. This evolved over the next few months into the foundation of the FIRE project. FIRE is one element of a proposed "modular" strategy of a series of smaller, defined scope experiments. The modular strategy spreads the technical risks and costs over a series of experiments compared to a single, integrated experiment. The FIRE device is shown in Figure 1.

The NSO study began in November, 1998 with a detailed review of the BPX-AT device. The BPX-AT study, which was performed in '91-'92, was undertaken in response to a Fusion Energy Advisory Committee (FEAC) finding that the then proposed BPX device, which had a major radius of $2.6 \mathrm{~m}$, and a cost of \$1.49 B (in FY 92 \$) was incompatible with budget realities. The proposed new device, which was named BPX-AT (advanced tokamak) had a much smaller major radius of $2.0 \mathrm{~m}$, and a much smaller cost of $\$ 642 \mathrm{M}$ (in FY 92 \$) [ref. 1]. BPX-AT was chosen as the starting point for the FIRE design because, like FIRE, its major

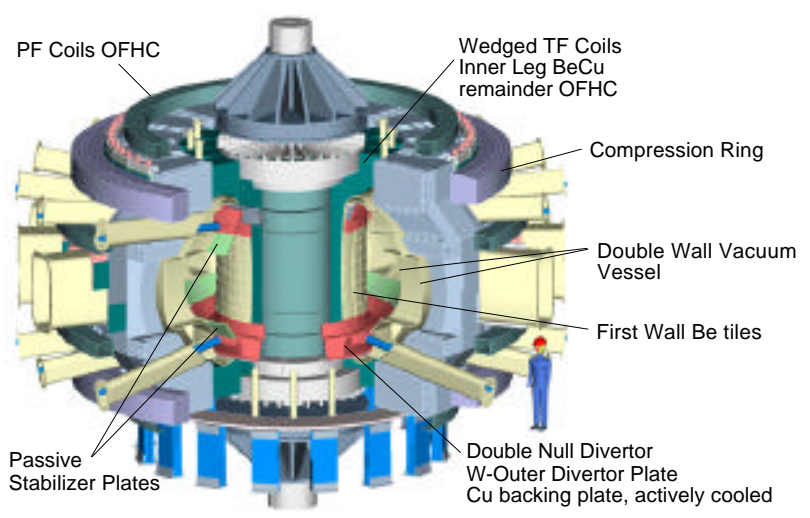

Coils cooled to $77^{\circ} \mathrm{K}$ prior to pulse

Figure 1. The FIRE Tokamak

goals were to achieve moderately high values of $\mathrm{Q}$ at the lowest possible cost. Like FIRE, it envisioned a phased mission which would minimize initial costs and would add features and elements as the program progressed and the additional features were required.

\section{FIRE'S PHYSICS REQUIREMENTS AND DEVELOPMENT OF MACHINE REQUIREMENTS}

The fundamental physics requirement chosen for FIRE is to attain high gain $(\mathrm{Q}>10)$. This would permit FIRE to determine the conditions required to achieve alphadominated plasmas. This includes energy confinement scaling, $\beta$-limits, and density limit scaling with alpha dominated heating. Another important goal is to achieve these objectives at a cost of less than \$1B.

Schultz [1] performed parametric design studies using a new spreadsheet he developed called FIRESALE. This spreadsheet permits $\mathrm{R}_{0}, \mathrm{~A}, \mathrm{~B}_{\mathrm{t}}$, and $\mathrm{q}_{\mathrm{lim}}$ to be varied and projects approximate machine costs using costing algorithms. These studies led to the adoption of the FIRE "baseline" machine parameters given in Table 1, which also compares the parameters of the BPX-AT.

\section{ENGINEERING ADVANCES INCORPORATED INTO FIRE}

Advances in tokamak engineering since the BPX-AT study have been adopted for FIRE in the divertor, vacuum vessel, plasma control, and machine configuration areas. FIRE has also benefited from advances in engineering analysis, design, and systems engineering. All of these have helped to improve FIRE's performance and costs which are still being evaluated. 
Table 1. Baseline FIRE Machine Parameters and Comparison to BPX-AT

\begin{tabular}{|l|l|l|}
\hline Parameter & FIRE & BPX-AT \\
\hline $\mathrm{R}_{0}$, major radius & $2.0 \mathrm{~m}$ & $2.0 \mathrm{~m}$ \\
\hline $\mathrm{A}$, minor radius & $0.525 \mathrm{~m}$ & $0.5 \mathrm{~m}$ \\
\hline $\mathrm{B}_{0}$, field on axis & $10 \mathrm{~T}$ & $10 \mathrm{~T}$ \\
\hline $\mathrm{Q}$ & $\sim 10$ & $\sim 5$ \\
\hline $\mathrm{I}_{\mathrm{p}}$ & $6.5 \mathrm{MA}$ & $6.25 \mathrm{M}$ \\
\hline Fusion power & $200 \mathrm{MW}$ & $150 \mathrm{MW}$ \\
\hline Flat top time, $\mathrm{s}$ & $18.5^{1} \mathrm{~s}$ & $10 \mathrm{~s}$ \\
\hline $\begin{array}{l}\text { Triangularity, } \\
\delta_{95 \mathrm{~m}} \delta_{\mathrm{x}}\end{array}$ & $0.4 ; 0.7$ & $0.25 \quad$ to $0.35 ;$ \\
\hline $\begin{array}{l}\text { Elongation, } \\
\kappa_{95}, \kappa_{\varepsilon}\end{array}$ & $1.8 ; 2.0$ & 0.40 to 0.65 \\
\hline
\end{tabular}

\section{A. Divertors}

FIRE will utilize gaseous (radiative) divertors like those proposed for TPX [2]. The divertor components are shown in Fig. 2 [3]. The outer divertor module, which receives $80 \%$ (34.3 MW) of the power, is actively cooled. It builds on fabrication technologies developed for the ITER divertor. The module incorporate tungsten-brush armor

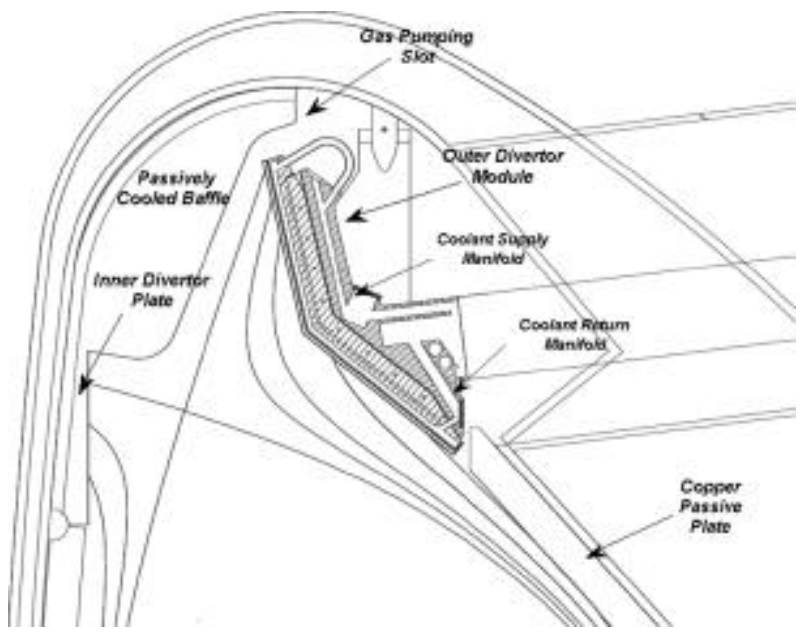

Figure 2. FIRE Divertor components

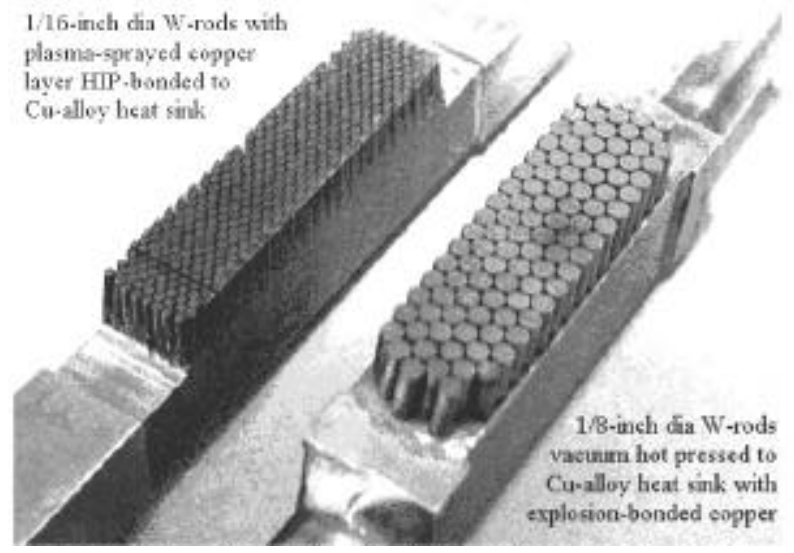

Figure 3. Two Variants of Tungsten Brush Armor Proposed for FIRE

similar to that shown in Fig. 3. This armor has been tested at Sandia Laboratory at $25 \mathrm{MW} / \mathrm{sq}$. m for 1000 cycles. It consists of 24 modular copper alloy finger plates that are attached to a support structure. The inner divertor plate receives only $20 \%$ of the divertor power. It consists of inertially cooled beryllium coated copper. The baffle is similar in construction and may be joined to the inner divertor plate to simplify maintenance.

\section{B. Vacuum Vessel and Plasma Control Coils}

FIRE proposes to use a double walled vessel with integral shielding, in-vessel copper passive stabilizer coils, and invessel active control coils which are similar to those first proposed in the TPX device. The FIRE vessel and control

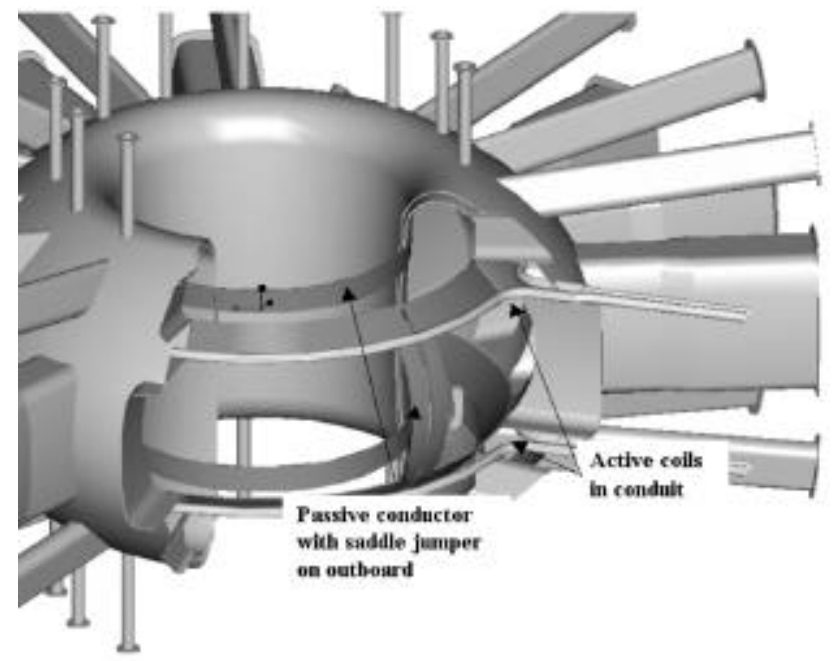

Figure 4. FIRE's Double Walled Vacuum Vessel, InVessel Passive Plates, and Active Control Coils

coils are shown in Fig. 4 [4].

This configuration has many advantages:

- it forms a stiffer, stronger vessel which is better able to resist disruption loads;

- it reduces the nuclear heating of the TF and PF coils;

- $\quad$ it reduces the dose rate to the electrical insulation of the PF coils and for much of the TF coil area;

- $\quad$ it reduces activation of machine components and makes possible "hands on" maintenance of components external to the vessel.

- $\quad$ it can make use of the remote welding processes developed as part of the ITER R\&D program.

The plasma passive and active control coil design has greatly benefited from the advances made in plasma stabilization and position control for TPX [ref. 3] and, more recently, KSTAR [ref. 4]. The copper passive stabilizers are in a close-fitting saddle configuration. Their closeness improves their effectiveness in damping the rapid vertical instabilities in the plasma. The saddle configuration (with zero net turns) minimizes effects on plasma start up. The design of the active control coils is similar to those of TPX and KSTAR, but with one significant new feature: their location in the vessel jacket, construction in quadrants (same as the vessel), and redundant turn segments. Their closeness to the plasma 
(compared to using external coils) improves their effectiveness and reduces their power requirements. Their in-vessel location better protects them, and frees up space in the vessel to improve the assembly, maintainabity and future upgrade options of the in-vessel components.

\section{Structural Configuration}

One of the first issues that the FIRE project focused on because of its broad impact on performance and costs was the selection of the structural configuration of the device. FIRE has benefited considerably from a variety of projects which have studied the various structural configurations for a tokamak. Wedged (example: BPX, [ref. 5]), bucked (example: ITER [ref. 6] ), and combined bucked / wedged (example: IGNITOR [ref. 7] and Alcator C-Mod) TF configurations were all considered. At the conclusion of this detailed study a wedged configuration was chosen. Each concept has unique benefits, but in making the selection, significant weighting was given to reaction of torques imposed on the TF coils and the desire to avoid keys to keep costs low. Torque reaction is one of the most challenging engineering areas for tokamaks with highly shaped plasmas and "external" PF coils. These torques are due to the fields from the central solenoid and PF coils crossing the inner leg of the TF coil. In FIRE the wedge

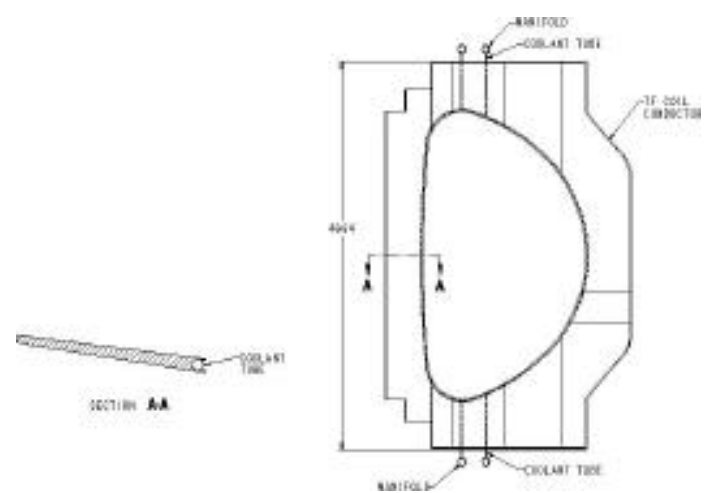

Figure 5 (a) A TF coil turn showing cooling and plate details.

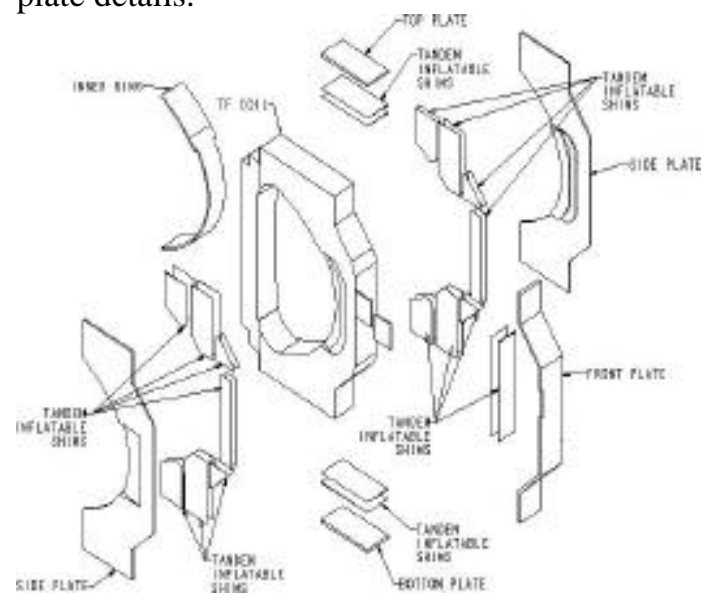

Figure 5 (b) FIRE TF Coil / Case Assembly Details pressure is high enough to react these loads by the frictional forces developed between the insulated turns. The maximum calculated shear in the inner leg at the midplane is $\sim 50 \mathrm{MPa}$. Using a conservative coefficient of friction of 0.3 and the calculated wedging pressure of $\sim 200$ $\mathrm{MPa}$, the allowable stress is $60 \mathrm{MPa}$. giving a factor of safety of 1.2

The decrease in wedge pressure at the top and bottom of the inner leg due to the outwards loads on the horizontal legs is often a problem in wedged machines. To counteract this effect, FIRE uses large compression rings around the $\mathrm{TF}$ coils to provide radially inwards forces to partially offset them; this approach is used by IGNITOR and BPX. An advantage of this arrangement is that the radial load increases during a pulse as the copper heats and expands against the cold rings and coil cases.

\section{TF Coils}

FIRE uses partially cased liquid nitrogen cooled copper coils. The design of the coils is similar to those of BPX, but with several differences which are chosen to reduce costs. An exploded view showing the details of a FIRE TF coil is shown in Fig. 5. The BPX coil cases had integral "box sections" in the rear portions which were welded together during assembly to form a continuous structure which performed a function similar to the TF compression rings on FIRE. On FIRE, the TF coil cases are not welded to each other and the compression rings are a separate structure. This simplifies assembly and reduces assembly time and cost. The other area of difference is in the fabrication of the coil turns. The BPX TF coil turns were to be fabricated of a high strength, high conductivity variant of $\mathrm{C} 17510$ beryllium copper (BeCU) developed for BPX by Brush-Wellman, Inc. [9]. Each turn is fabricated from four pieces of $\mathrm{BeCu}$ joined by welding followed by -heat treating. FIRE, in comparison, will use the $\mathrm{BeCu}$ only in the inner leg where its higher cost is justified by the need for its strength, and will use oxygen free copper in the remainder. This reduces the magnet cost, and reduces power and cryogenic consumption. Two processes are being investigated for joining the plates: electron beam welding or possibly by a new process called friction stir welding (FSW) [10]. Both processes minimize the heat affected zone and therefore minimize effects on copper near the joint.

FIRE's electrical insulation scheme is similar to that proposed for BPX. Formed cuffs of pre-cured polyimide-glass will be used to insulate the edges of the turns. Pre-cured sheets of polyimide-glass sheets will be used for the turn to turn insulation; bonding is not planned, since the frictional load capacity due to wedge pressure is adequate. The coils will be ground wrapped with B-stage glass/polyimide insulation and press cured. The peak radiation dose at the insulation is $1.44 \times 10^{10}$ rads at the mid point of the inner leg. This is within 
reasonable limits for polyimide resins [11]. A possible option for the insulation is hybrids of epoxy and polyimide which are currently being developed. They are expected to be suitable for high radiation levels but are easier to process [12] . FIRE will use tandem stainless steel pillow shims similar to those proposed for BPX. They will be inflated with polyimide / silica grout to position the coil in its case and transfer loads. One set of shims will be used at assembly, with the second reserved as a back up.

\section{E. CS and PF Coils}

The FIRE CS coils are similar in construction to the BPX coils; however, they will be made of OFHC rather than $\mathrm{BeCu}$. The CS coils will be fabricated from copper discs that are water jet cut in a spiral. Layer to layer joints will be made by electron beam welding. The discs will be "sprung" to permit the B-stage glass / epoxy turn insulation and a glass / epoxy turn to turn barrier strips to be applied' The stacked discs will be separated by glass/epoxy discs with radial grooves for liquid nitrogen cooling. The PF coils will be similar in construction, but because of their larger size, will be tension wound using extruded copper bars.

\section{F. In-Vessel Remote Maintenance}

In-vessel remote maintenance will be performed by an articulated boom manipulator. The manipulator will transfer removed activated components to a mobile cask which will contain and transport the components to a hot cell for servicing. Modular designs are being used to the greatest extent possible.

\section{Expanded Mission Capabilities}

Analyses performed during the study indicate that the TF and PF coils have the capability of operating in several modes in addition to the baseline [13]:

- $\quad$ high $\mathrm{Q}$ mode (at $\mathrm{B}_{\mathrm{T}}=10 \mathrm{~T} ; \mathrm{I}_{\mathrm{P}}=6.44 \mathrm{MA} ; 21 \mathrm{~s}$ flat top with D-T; 31 s flat top with D-D);

- a "TPX-like" advanced physics mode $\left(\right.$ at $\mathrm{B}_{\mathrm{T}}=4 \mathrm{~T} ; \mathrm{I}_{\mathrm{P}}=2$ MA; 250 s flat top) and

- a high $\mathrm{Q}$ advanced physics mode at $\mathrm{B}_{\mathrm{T}}=8 \mathrm{~T} ; \mathrm{I}_{\mathrm{P}}=5$ MA; 44 s flat top).

Another possible option is to use the higher strength variant of $\mathrm{BeCu} \mathrm{C17510} \mathrm{with} \mathrm{a} \mathrm{conductivity} \mathrm{of} 68 \%$ rather than the $77 \%$ currently used in the baseline. This would permit operation at $12 \mathrm{~T}$ and $7.7 \mathrm{MA}$ with a $12 \mathrm{~s}$ flat-top in D-T. The flat-top times in the other modes would be reduced by $\sim 12 \%$ due to the lower conductivity. Studies are planned to determine the impact of extended pulse operation on the divertor and first wall.

\section{Near-Term Plans}

Detailed costing is underway and will be the major effort for the upcoming year. The cost breakdown is expected to be roughly $1 / 3$ for the tokamak (TF and PF coils, vacuum vessel, structure), 1/3 for power supplies, diagnostics, heating systems, and remote maintenance (with the power supplies being the dominant cost item in this category), and $1 / 3$ for facilities and siting. Consequently, power supplies, facilities and siting are seen as important areas that will be focused on to keep costs as low as possible. Also planned are continued studies of FIRE's long pulse, AT capabilities and more detailed analyses of disruption loads and thermal stresses induced in the vessel by nuclear heating.

\section{Summary}

The FIRE study started with a review of BPX-AT, utilized parametric studies to set an initial design point, and, based on these, developed an initial integrated design. To keep costs low and performance high, FIRE extensively sought and, where possible, incorporated attractive features from other machines and studies and new materials and processes. The resulting design meets the requirements set for it $\left(\mathrm{B}_{\mathrm{T}}=10 \mathrm{~T} ; \mathrm{Q}=5-10\right.$; flat top time $\left.=10 \mathrm{~s}\right)$ and is also capable of long pulse, AT operation. Detailed costing is now underway. Efforts will be concentrated on the power supply, facilities, and siting which have high cost leverage.

\section{REFERENCES}

[1] "BPX-AT White Paper"; Princeton Plasma Physics Laboratory; February, 1992.

[2] W. Reiersen, "TPX Conceptual Design Overview"; Princeton Plasma Physics Laboratory, March, 1993.

[3] B. Nelson, et. al; "fusion Ignition Research Experiment Vacuum Vessel Design and Integration"; IEEE/NPSS Symposium on Fusion Engineering, Albuquerque, NM; October, 1999

[4] "KSTAR TSER Presentations"; Korea Basic Science Institute, December, 1997.

[5] P. Heitzenroeder, "BPX TF Coil Design"; IEEE/NPSS Symposium on Fusion Engineering, October, 1991.

[6] P. Titus, "ITER EDA Out-Of-Plane Structural Design and Analysis; IEEE/NPSS Symposium on Fusion Enginering; October, 1993.

[7] P. Titus, "Structural Behavior of IGNITOR"; US-IGNITOR Working Group Meeting, November, 1998.

[8] "Fire Feasibility Study"; FIRE Project Report No. 81_991006_FIREStudy_FT.doc

[9] Holt Murray, et. al; "Fracture Testing and Performance of Beryllium Copper Alloy C17510"; American Society for Testing of Materials, Std. Tech. Pub. 1184.

[10] A. Berger and E. Peterson, "Cost Estimate for TF/ PF Coils"; Advanced Energy Systems, September, 1999.

[11] M.E. Sawan and H.Y. Khater, "Initial Nuclear Performance Evaluation of the FIRE Ignition Device"; IEEE/NPSS Symposium on Fusion Engineering, October, 1999.

[12] M.L. Tupper, et. al, "Magnet Insulation with Resistance to High Levels of Radiation"; Proceedings of the $16^{\text {th }}$ International Conference on Magnet Technology, Jacksonville, Fl.; September, 1999.

[13] P. Titus, " FIRE/NSO Toroidal Field Coil Structural / Thermal Analyses"; IEEE/NPSS Symposium on Fusion Engineering; October, 1999. 